\title{
Impact of a single, short morning bright light exposure on tryptophan pathways and visuo- and sensorimotor performance: a crossover study
}

\author{
Wolfgang Schobersberger ${ }^{1,2^{*}}$, Cornelia Blank ${ }^{1}$, Friedrich Hanser ${ }^{3}$, Andrea Griesmacher $^{4}$, Markus Canazei ${ }^{5}$
} and Veronika Leichtfried ${ }^{1}$

\begin{abstract}
Background: Bright light (BL) has been shown to be effective in enhancing both cognitive and physical performances. Alterations in nighttime melatonin levels have also been observed. However, evaluations of light-induced changes in the preceding biochemical processes are absent. Therefore, the impact of a single morning BL exposure on sensorimotor and visuomotor performance, as well as tryptophan (trp) and trp metabolites, was evaluated in this study.

Methods: In a crossover design, 33 healthy volunteers were randomly exposed to $30 \mathrm{~min}$ of $<150 \mathrm{~lx}$ at eye level (office light, OL) and $5000 \mathrm{~lx}$ at eye level (bright light, BL) of $6500 \mathrm{~K}$ in the morning hours. Trp, sulfatoxymelatonin (aMT6s), and kynurenine (kyn) courses over the morning hours were analyzed, and changes in sensori- and visuomotor measures were examined.

Results: Motoric performance increased in both setups, independent of light intensity. aMT6s and kyn decreased equally under both lighting conditions. Trp levels decreased from a mean (95\% confidence interval) of 82.0 (77.2-86.9) to 66.5 (62.5-70.1) in the OL setup only.

Conclusion: These data suggest that BL in the morning hours has a limited effect on visuo- and sensorimotor performance. Nevertheless, trp degradation pathways in the morning show diverse courses after $\mathrm{OL}$ and $\mathrm{BL}$ exposure. This suggests that trp courses can potentially be altered by BL exposure.
\end{abstract}

Keywords: Bright light, Sensorimotor performance, Visuomotor performance, Tryptophan, Melatonin, Kynurenine

\section{Background}

Even without environmental stimuli, both cognitive and physical performance vary in an iterative rhythm lasting approximately $24 \mathrm{~h}$. Especially in the morning hours, performance is reduced $[1,2]$. However, optimized performance and productivity are often desired regardless of the time of the day (i.e., for sport competitions and in the work environment). Therefore, a demand for performance-enhancing strategies exists.

\footnotetext{
* Correspondence: wolfgang.schobersberger@tirol-kliniken.at

'Institute for Sports Medicine, Alpine Medicine and Health Tourism, UMIT -

University for Health Sciences, Medical Informatics and Technology, Eduard

Wallnöfer Zentrum 1, 6060 Hall in Tyrol, Austria

${ }^{2}$ Tirol Kliniken GmbH, Anichstraße 35, 6020 Innsbruck, Austria

Full list of author information is available at the end of the article
}

The suprachiasmatic nuclei $(\mathrm{SCN})$ play an important role in diurnal variations of mental and physical performance and strongly determine productivity and errorsusceptibility [3, 4]. SCN activity can be modulated by exogenous parameters, e.g., sleep-wake patterns, meals, activity, or light exposure.

Light plays an essential role in modulating the circadian system either by direct effects or phase-shifting effects on the circadian clock. Optimized indoor illumination and bright light (more than $1000 \mathrm{~lx}$ at eye level) were shown to be of value for health and work efficiency strategies, inducing positive effects on mood and increasing activation [5-8], as well as cognitive and exercise performance [9-12].

(c) The Author(s). 2018 Open Access This article is distributed under the terms of the Creative Commons Attribution 4.0 International License (http://creativecommons.org/licenses/by/4.0/), which permits unrestricted use, distribution, and reproduction in any medium, provided you give appropriate credit to the original author(s) and the source, provide a link to the Creative Commons license, and indicate if changes were made. The Creative Commons Public Domain Dedication waiver (http://creativecommons.org/publicdomain/zero/1.0/) applies to the data made available in this article, unless otherwise stated. 
One underlying mechanism might be light's ability to influence levels of hormones that vary approximately with a period length of $24 \mathrm{~h}$, such as melatonin and its precursors [13-15]. Tryptophan (trp) is the basic molecule that can be converted to serotonin and further degraded to melatonin [16]. Advancement of the circadian phase via morning bright light (BL), in combination with afternoon melatonin uptake, recently proved successful [17]. Numerous studies have reported the impact of light exposure on melatonin production and excretion, with an emphasis on its relevance for cognitive and physical performance $[18,19]$. Beyond acute nighttime suppression of melatonin, there are several studies regarding the impact of $\mathrm{BL}$ on alertness [1, 20], as well as cognitive $[18,21,22]$ and physical performance [22-27]. However, research specifically focusing on the potential consequences of acute BL application on sensorimotor (coordination) and visuomotor (reaction) performance is lacking. Moreover, research on potential underlying biochemical mechanisms of $\mathrm{BL}$, such as melatonin precursors and trp metabolites, known collectively as kynurenines (kyns), which are involved in a variety of biological activities (e.g., inflammation, immune response, exercise, mental health) [28], is scarce [29, 30]; addressing this gap could augment our understanding of the impact of $\mathrm{BL}$ on cognitive and physical performance and its biochemical pathways.

The aim of this study was twofold. First, we evaluated the impact of a single 30-min BL exposure in the early morning hours on visuomotor and coordinative performance in healthy adults. Second, we collected data on trp and trp metabolites (kyn) to assess the potential association between BL-induced changes in visuomotor and coordinative performance and changes in trp and trp metabolites. It was hypothesized (H1) that visuomotor and coordinative performance would improve after BL exposure. Furthermore, it was hypothesized (H2) that urine melatonin levels would be suppressed because of the morning BL exposure. Finally, we also hypothesized (H3) a significant negative association between changes in visuo- and sensorimotor performance and trp, as well as kyn.

\section{Materials and methods \\ Participants}

Via the Internet, 35 study participants between 18 and 45 years of age, from the Tirol Kliniken/University Hospital Innsbruck (Austria), the University for Health Sciences, Medical Informatics and Technology (UMIT, Hall, Austria), and the Tyrolean Confederation of Sports Shooters (Austria), were recruited. None of the participants were on medications, had ever worked in shifts, had taken a transmeridian flight in the previous 2 months, or suffered from chronic diseases. Moreover, all participants maintained their daily lifestyles including regular sleeping times at least 2 weeks before the study begins. For details, see Leichtfried et al. [20].

Additionally, respondents with depressive and/or sleeping disorders were excluded from the study. To evaluate depression and depressive symptoms, the Hospital Anxiety and Depression Scale (German version; HADS) was used by applying a cut-off value $>8$ as the exclusion criterion [31]. Sleep quality was determined via the Pittsburgh Sleep Quality Index (PSQI) by applying a cut-off value $>5$ as the exclusion criterion [32]. All participants gave written informed consent prior to participation in the study.

\section{Experimental trials}

A prospective, explorative crossover design with a washout phase of 7 days was implemented. In order to control for natural daylight, all investigations were performed in the winter months (15 November-25 February). On both test days, the participants slept at their homes, woke at 6:00 a.m., collected their first morning urine samples (all participants were instructed how to provide urine samples), and had their habitual breakfast. There was no instruction regarding a standardized breakfast, but all participants were told to follow their usual morning routine. Participants arrived at the study location at 7:00 a.m. and brought their first morning urine samples along with them. During the study period, the sun had not risen at the start of the indoor protocol at 07:05 a.m. (November 15: 07:18 a.m.; December 1: 07:40 a.m.; January 1: 08:01 a.m.; February 1: 07:41 a.m.; February 25: 07:07 a.m.), thus guaranteeing that bright light exposure by sun light had not affected the volunteers at the start of the indoor protocol at 07: 05 a.m. On two occasions, according to an interval of 7 days, each study participant was exposed to light for 30 min starting at 07:40 a.m. One of the exposures was to $\mathrm{BL}$ and the other to office light (OL). Before and after the light exposures, participants stayed in the same room, illuminated by normal workplace light $(<100 \mathrm{~lx}$ at eye level, 4000 Kelvin). In total, 18 of the participants were randomly assigned to receive the $\mathrm{BL}$ exposure first, followed by the OL exposure 7 days later, and the other 17 participants were assigned to receive the OL exposure first, followed by the BL exposure 7 days later. Identical experimental procedures on both days of exposure were applied (Table 1).

\section{Light exposures}

Exposure to BL was performed in a light cabin [33], which was equipped with fluorescent lamps (OSRAM $58 \mathrm{~W} / 965$ Biolux, $3700 \mathrm{~lm}$ ) with a light intensity (illuminance) of 5000 lux at eye level, a color 
Table 1 Experimental procedure on both study days

\begin{tabular}{|c|c|c|}
\hline $\begin{array}{l}\text { Time of day } \\
\text { (a.m.) }\end{array}$ & Activity & $\begin{array}{l}\text { Light } \\
\text { exposure }\end{array}$ \\
\hline 06:00 & Wake up (morning urine collection) & $\begin{array}{l}\text { Residential } \\
\text { light }\end{array}$ \\
\hline 07:00 & Arrival at the study location & Workplace \\
\hline 07:05 & Urine sampling I, blood sampling I & Workplace \\
\hline 07:15 & VAS pre (data not shown, [26]) & Workplace \\
\hline 07:20 & $\begin{array}{l}\text { Visuo- and sensorimotor performance } \\
\text { tests I }\end{array}$ & Workplace \\
\hline 07:40-08:10 & Light exposure & $\mathrm{BL}$ or $\mathrm{OL}$ \\
\hline 08:12 & VAS post (data not shown, [26]) & Workplace \\
\hline 08:15-09:00 & $\begin{array}{l}\text { Sustained attention test } \\
\text { (data not shown, [26]) }\end{array}$ & Workplace \\
\hline 09:05 & Urine sampling II, blood sampling II & Workplace \\
\hline 09:15 & $\begin{array}{l}\text { Visuo- and sensorimotor performance } \\
\text { tests II }\end{array}$ & Workplace \\
\hline 11:05 & Urine sampling III, blood sampling III & Workplace \\
\hline
\end{tabular}

VAS visual analogue scale, pre before light exposure, post after exposure to either bright light (BL, 5000 lux, $6500 \mathrm{~K}$ ) or office light $(\mathrm{OL},<150$ lux, $6500 \mathrm{~K}$ ), workplace light exposure $<100$ lux, $4000 \mathrm{~K}$

temperature of $6500 \mathrm{~K}$, and a mean field of view luminance of $1500 \mathrm{~cd} / \mathrm{m}^{2}$. Without fixing the head and gaze of the subjects, corneal light levels could be kept constant in this cabin.

The photometrical specification of the OL setup, again performed in the light cabin, was chosen to be similar to conventional office illumination, with a light intensity of $<150$ lux at eye level, and a mean field of view luminance of $85 \mathrm{~cd} / \mathrm{m}^{2}$.

Table 2 summarizes relevant photometrical measures of both lighting scenarios according to the recommendations from Lucas et al. [34]. In terms of melanopic illuminance levels, BL was more than 33 times brighter than OL. For further specifications of the light cabin, see Leichtfried et al. [33].

Table 2 Photometrical measures of the light exposures

\begin{tabular}{lll}
\hline Photometrical measures [unit] & Office light (OL) & Bright light (BL) \\
\hline $\begin{array}{lll}\text { Corneal intensity-related measures } \\
\text { Photopic illuminance [lux] }\end{array}$ & $150 \pm 32$ & $5000 \pm 455$ \\
Irradiance $\left[\mu \mathrm{W} / \mathrm{cm}^{2}\right]$ & 59.18 & 1972.53 \\
Photon flux [1/ $\left.\mathrm{cm}^{2} / \mathrm{s}\right]$ & $1.61 \times 10^{14}$ & $5.37 \times 10^{15}$ \\
Photopigment-related measures (a-opic lux) [34] at mean illuminance levels \\
Cyanopic illuminance [lux] & 160.73 & 5357.65 \\
Melanopic illuminance [lux] & 152.88 & 5096.14 \\
Rhodopic illuminance [lux] & 154.17 & 5138.83 \\
Chloropic illuminance [lux] & 153.19 & 5106.38 \\
Erythropic illuminance [lux] & 147.36 & 4912.04 \\
\hline
\end{tabular}

Illuminance values: mean \pm standard deviation
Participants were instructed to keep their eyes open during $\mathrm{OL}$ and $\mathrm{BL}$ exposure. They were allowed to read newspapers, which were supplied by the authors.

Motor performance measures and blood parameters were recorded under standard workplace lighting from ceiling luminaires (equipped with fluorescent lamps; OSRAM 36W 840 Lumilux) with a light intensity of < 100 lux and a correlated color temperature of $4000 \mathrm{~K}$ at eye level of the study participants.

\section{Evaluation of chronotypes}

Since the individual chronotype might influence the study outcomes, chronotypes of the participants were evaluated at the beginning of the study using the MorningnessEveningness Questionnaire (MEQ) [35]. Analyses of biochemical parameters were controlled for chronotype.

\section{Physiological measures}

Urinary 6-sulfatoxymelatonin (aMT6s) was assayed using a commercially available competitive immunoassay kit (Buehlmann 6-SMT ELISA, Buehlmann Laboratories AG, Schoenenbuch, Germany) with a lower detection limit of approximately $0.5 \mathrm{pg} / \mathrm{mL}$ (for details, see [5]). All aMT6s analyses were done at the Central Institute for Medical and Chemical Laboratory Diagnostics, Innsbruck, Austria, and creatinine (crea)-standardized to account for differences arising from variations in urine concentrations.

Serum trp and kyn concentrations were measured by high-pressure liquid chromatography (HPLC) using 3-nitro-L-tyrosine as an internal standard [36]. Reference values for healthy subjects are $67.4 \pm 10$. $2 \mu \mathrm{mol} / \mathrm{L}$ for trp and $1.78 \pm 0.42 \mu \mathrm{mol} / \mathrm{L}$ for kyn [37].

The following three physiological parameters were subjected to statistical analyses in the present study: urinary 6-sulfatoxymelatonin normalized for crea (aMT6s/crea), trp, and kyn.

\section{Performance tests \\ Visuomotor reaction time analyses}

Reaction tests were performed using specifically designed equipment (Dr. Wieser GmbH, Salzburg, Austria) with four sensing devices, one for each hand and foot. Sixty random signals were transmitted to a rectangular black board (positioned in front of the participant) equipped with four red light-emitting diodes (LEDs) positioned on each corner of the black board. The participants were allowed to individually position the sensing devices (foot and hand switches) and were instructed to react as quickly as possible by touching the appropriate sensing device (top right-right hand, top left-left hand, bottom right-right foot, bottom left-left foot), depending on which LED flashed red. In addition to the total reaction time (TRT), the following four performance measures were recorded: number of correct reactions 
(CR), number of false reactions (FR), reaction time of correct reactions (RTCR), and reaction time for false reactions (RTFR). A test phase lasting $1 \mathrm{~min}$ was performed prior to the initial test.

\section{Sensorimotor (balance) test}

Balance tests were performed on an unstable surface (MFT Challenge Disk, MFT-Multifunktionale Trainingsgeräte $\mathrm{GmbH}$, Guntramsdorf, Austria). Threedimensional deviations from the horizontal zero position were evaluated using acceleration/inertial sensors. Sensors measure deviations from the horizontal plane, expressed as $A_{z}$, reflecting the extent to which the unstable surface is tilted. Values increase with increasing deviation from the horizontal plane, and low $A_{z}$ values reflect a "stable" balance.

A test phase of 1 min preceded every trial. After the test phase, the participant stepped off the unstable disc for $2 \mathrm{~min}$ and stepped on again for the actual recording phase, which lasted 2 min.

Balance tests were performed before and after light exposures, directly after the visuomotor reaction time analyses.

\section{Statistical analyses}

The distribution of the data was evaluated using a graphical approach (boxplots). Parametric tests were used for normally distributed data and non-parametric tests for skewed data (aMT6s/crea).

Differences between the three chronotypes, for changes over time ( $\Delta$ pre-post), were evaluated via the Kruskal-Wallis test for unmatched samples.

The statistical approach was chosen according to recommendations for cross-over designs [38]: to eliminate possible carry-over effects, an initial test was done. The results for both study periods were calculated and added up, followed by an unpaired $t$ test for the different sequence groups. A non-significant result indicates no carry-over effects. Treatment effects were calculated by comparing the respective differences between the measurement time points by performing an unpaired $t$ test for the two sequence groups. For aMT6s/crea data, a Mann-Whitney $U$ test was applied for both tests.

Regarding the biochemical parameters, changes over time in the two setups were calculated via linear models for repeated measurements and Friedman ANOVA (aMT6s/crea), including an interaction term (time $\times$ light). Dependent on the data distribution, the locations of possible changes were evaluated via $t$ test and Wilcoxon's test for paired samples (aMT6s/crea), with the Bonferroni correction applied.

To test for associations between changes in performance and biochemical parameters, Pearson (non-skewed data) or Spearman (skewed data) correlation analysis was performed as applicable. Even though data from four time points were recorded, deltas of change resemble the difference between biochemical parameters in the first and final urine sample taken. Effect sizes were presented as correlation coefficients, $r$, which followed Cohen's convention for effect size classification.

Results from categorical variables were reported as proportions, and continuous variables were reported as means \pm standard deviation (SD) or means and 95\% confidence intervals (CI). All statistical analyses were performed using SPSS (Statistical Package for Social Sciences, ver. 20.0; SPSS Inc., Chicago, IL, USA). All statistical tests were performed at a 0.05 level of significance.

\section{Results \\ Participants}

Thirty-three participants (16 males, 17 females) with an average age of $33.0 \pm 7.2$ years were included in the analyses. Two volunteers were excluded during the study because of acute infections. The participants showed a mean PSQI score of $3.8 \pm 1.8$ and a sleep efficiency of $85 \%$; no participant reported taking soporifics in the 4 weeks prior to the first light exposure. With respect to chronotype, $15.2 \%$ (5) of the participants were morning type, $12.1 \%$ (4) were evening type, and $72.7 \%$ (24) were moderate chronotype subjects. The results of the HADS-D indicated that 31 (93.9\%) of the participants were not depressed (score $<7$ ) and $2(6.1 \%)$ of the participants showed signs of slight depression (score 8-10). Chronotype-dependent differences in change scores ( $\Delta$ pre-post) were detected for aMT6s values only $(p=.009)$; thus, such differences were further considered only for aMT6s values.

\section{Performance data \\ Visuomotor performance}

Carry-over effects for all parameters could be ruled out (CR, $p=.862$; TRT, $p=.193$; RTCR, $p=.183$; RTFR, $p$ $=.795 ; \mathrm{FR}, p=.862$ ). TRT ranged from 0.46 to $0.88 \mathrm{~s}$. No changes in RTCR were detected at either light intensity. After both light exposures, CR was significantly higher and FR significantly lower as compared to before light exposure. No effects of light intensity were detected for any of the reaction parameters. Details are outlined in Table 3.

\section{Sensorimotor performance}

No carry-over effects were detected $(p=.884)$. Deviations from the horizontal plane $\left(A_{z}\right)$ ranged from 0 . 21 to $1.58 \mathrm{~m} / \mathrm{s}^{2}$, with mean values of $0.52 \pm 0.21 \mathrm{~m} / \mathrm{s}^{2}$. Under DOL, $A_{z}$ decreased from $0.60 \pm 0.27$ to $0.47 \pm 0$. $18 \mathrm{~m} / \mathrm{s}^{2}(p=.028) . A_{z}$ also decreased from $0.53 \pm 0.17$ to $0.47 \pm 0.17 \mathrm{~m} / \mathrm{s}^{2}$ after BL exposure $(p=.005)$. No effects of light intensity on balance performance could be found $(p=.275)$. Details are outlined in Table 3. 
Table 3 Results of performance tests before and after office light (OL) and bright light (BL) exposure

\begin{tabular}{|c|c|c|c|c|c|c|c|}
\hline & \multicolumn{3}{|c|}{ OL exposure } & \multicolumn{3}{|c|}{ BL exposure } & \multirow[t]{2}{*}{$p$ value $(\mathrm{OL}$ vs. $\mathrm{BL}$} \\
\hline & Before & After & $p$ value & Before & After & $p$ value & \\
\hline \multicolumn{8}{|c|}{ Visuomotor performance } \\
\hline$C R[n]$ & $55.1 \pm 4.3$ & $56.6 \pm 2.6^{*}$ & 0.021 & $55.6 \pm 3.0$ & $56.7 \pm 2.0^{*}$ & 0.033 & 0.739 \\
\hline TRT [s] & $0.63 \pm 0.09$ & $0.64 \pm 0.08$ & 0.803 & $0.63 \pm 0.08$ & $0.63 \pm 0.08$ & 0.975 & 0.959 \\
\hline RTCR [s] & $0.64 \pm 0.09$ & $0.64 \pm 0.08$ & 0.843 & $0.64 \pm 0.08$ & $0.64 \pm 0.08$ & 0.706 & 0.813 \\
\hline RTFR [s] & $2.45 \pm 1.62$ & $1.96 \pm 0.37$ & 0.223 & $2.16 \pm 0.64$ & $2.32 \pm 1.54$ & 0.626 & 0.128 \\
\hline $\mathrm{FR}[n]$ & $4.9 \pm 4.3$ & $3.4 \pm 2.6^{*}$ & 0.021 & $4.4 \pm 3.0$ & $3.3 \pm 2.0^{*}$ & 0.033 & 0.739 \\
\hline \multicolumn{8}{|c|}{ Sensorimotor performance } \\
\hline $\mathrm{Az}\left[\mathrm{m} / \mathrm{s}^{2}\right]$ & $0.60 \pm 0.27$ & $0.47 \pm 0.17^{*}$ & 0.028 & $0.53 \pm 0.17$ & $0.47 \pm 0.18^{*}$ & 0.005 & 0.275 \\
\hline
\end{tabular}

Values represent mean values \pm standard deviations

$O L$ office light, $B L$ bright light, $A_{z}$ deviation from horizontal plane, $C R$ correct reactions, $T R T$ total reaction time, $R T C R$ reaction time correct reactions, $R T F R$ reaction time false reactions, $F R$ false reactions

${ }^{*} p<0.05$ compared to before light treatment

\section{Biochemical data}

All biochemical data are shown in Table 4. No carry-over effects were detected for any biochemical parameter.

\section{aMT6s/crea}

The average aMT6s/crea baseline values in both light groups were comparable, with 20.8 (CI 12.2-29.4) ng/mg in the OL group and $14.0(\mathrm{CI} 8.6-20.5) \mathrm{ng} / \mathrm{mg}$ in the BL group ( $p=.542)$; aMT6s/crea changed significantly under both light setups (OL and BL $p<.001)$. Pairwise comparisons revealed that changes in both light setups occurred from morning urine to 9:15 a.m. as well as to 11:15 a.m. (p $<.001)$ and from 9:15 a.m. to 11:15 a.m. $(p<.001)$. In the BL setup, aMT6s values additionally decreased from 7:15 a. m. to 9:15 a.m. $(p=.015)$. We did not detect any timedependent effect of the two light intensities $\left(p_{1}=.717, p_{2}\right.$ $=.918, p_{3}=.654, p_{4}=.477, p_{5}=.172, p_{6}=.608$; with $p_{1}$ : morning urine to $7: 15$ a.m.; $p_{2}$ : morning urine to 9:15 a.m.; $p_{3}$ : morning urine to 11:15 a.m.; $p_{4}:$ 7:15 a.m. to 9:15 a.m.; $p_{5}:$ 7:15 a.m. to 11:15 a.m.; $p_{6}:$ 9:15 a.m. to 11:15 a.m.).

Changes in aMT6s/crea levels from pre- to post-light exposure in subjects with evening chronotype were greater $(35.76 \pm 24.01)$ compared to subjects with a moderate chronotype $(6.96 \pm 14.60 ; p=.002)$. No differences were found for morning versus evening type subjects $(p=.072)$ or morning versus moderate chronotype subjects $(p=.268)$.

\section{Trp}

Mean baseline trp levels (at 7:15 a.m.) were $82 \mu \mathrm{mol} /$ $\mathrm{L}$ (CI 77.2-86.9) for $\mathrm{OL}$ and $75.6 \mu \mathrm{mol} / \mathrm{L}$ (CI 70 . 3-81.0) for BL. The trp levels changed over time in the OL setup only $(p<.001)$. Changes occurred from 7:15 a.m. to 9:15 a.m. and from 7:15 a.m. to 11:15 a. m. $(p<.001$ at both times $)$. A significant light effect was only found for the period from 7:15 a.m. to 11: 15 a.m. ( $p=.045$; Fig. 1$)$.

\section{Kyn}

Mean baseline trp levels (at 7:15 a.m.) of $3.2 \mu \mathrm{mol} / \mathrm{L}$ (CI 2.8-3.7) for OL and $2.8 \mu \mathrm{mol} / \mathrm{L}$ (CI 2.5-3.0) for BL were measured. The kyn levels remained unchanged in both light setups (BL, $p=.288$; OL, $p=.070$ ). No differences in the scores between different time periods were detected between the two light setups.

Table 4 Sulfatoxymelatonin (aMT6s/crea), tryptophan (trp), and kynurenine (kyn) concentrations under office light (OL) and bright light (BL) exposure

\begin{tabular}{|c|c|c|c|c|c|c|}
\hline \multirow[t]{2}{*}{ Time } & \multicolumn{2}{|c|}{ aMT6s/crea [ng/mg] } & \multicolumn{2}{|l|}{$\operatorname{trp}[\mu \mathrm{mol} / \mathrm{L}]$} & \multicolumn{2}{|l|}{$\operatorname{kyn}[\mu \mathrm{mol} / \mathrm{L}]$} \\
\hline & OL exposure & BL exposure & OL exposure & BL exposure & OL exposure & $B L$ exposure \\
\hline Morning urine & $20.8(12.2-29.4)$ & $14.0(8.6-19.4)$ & n.a. & & n.a. & \\
\hline 7:15 a.m. & $17.8(10.9-24.7)$ & $14.6(8.7-20.5)$ & $82.0(77.2-86.9)$ & $75.6(70.3-81.0)$ & $3.2(2.8-3.7)$ & $2.8(2.5-3.0)$ \\
\hline 9:15 a.m. & $12.1(8.0-16.1)^{\#}$ & $12.2(7.3-17.1)^{\#, *}$ & $69.5(65.0-74.1)^{*}$ & $69.3(64.9-73.7)$ & $2.8(2.6-3.0)$ & $2.7(2.4-2.9)$ \\
\hline 11:15 a.m. & $8.4(2.1-14.7)^{\#, * * * * * *}$ & $3.9(2.4-5.4)^{\#, * * * * *}$ & $66.5(62.5-70.1)^{*}$ & $69.5(65.9-73.1)$ & $2.7(2.4-3.0)$ & $2.6(2.3-2.8)$ \\
\hline
\end{tabular}

Values represent mean values and $95 \%$ confidence intervals

${ }^{\#} p<0.05$ as compared to morning urine

${ }^{*} p<0.05$ as compared to $7: 15$ a.m.

${ }^{* *} p<0.05$ as compared to 9:15 a.m. 


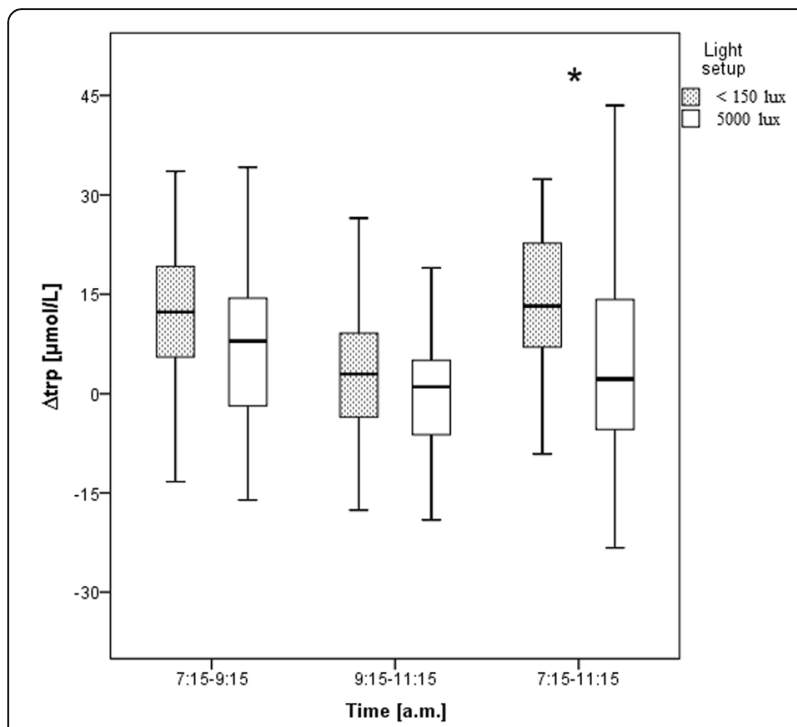

Fig. 1 Differences in tryptophan serum levels $(\Delta)$ over time for the two light setups (office light and bright light). ${ }^{*} p<0.05$ versus the other light setup

\section{Association between performance and biochemical measures}

Correlation analyses revealed that changes in kyn values were significantly associated with changes in TRT $(p=.032 ; \rho=0.280), \operatorname{RTCR}(p=.010 ; \rho=0.334)$, CR $(p=.049 ; \rho=0.257)$, and trp values $(p<.001 ; \rho=0.645)$.

\section{Discussion}

Early morning light exposure has been shown to improve a variety of physical and cognitive performance indices after sleep. We evaluated the impact of a single early morning BL exposure on performance and biochemical parameters. In addition to previously published data [20], the novel approach of this study was the quantification of a possible association between the impact of $\mathrm{BL}$ on the courses of trp and trp degradation products, melatonin and kyn, and motor performance parameters.

An exclusive effect of the BL intervention was found for trp data only, showing a lower decrease in trp values over time in the BL setup. With respect to the measured sensorimotor functions, participants reacted identically in both light setups, with better balance and choice reaction performance after both light exposures. Some of the changes in performance parameters were found to be associated with changes in biochemical parameters.

\section{Performance data}

According to the suggestion that most prominent [39] and immediate effects [40] of BL exposure on cognitive performance should be expected during the day when provided before midday, an early morning light regimen was chosen in the present design. However, the single early morning light intervention was not effective in increasing visuomotor performance. The present results are also in contrast to those from a study conducted during the Antarctic winter, where BL was effective in improving alertness and cognitive performance [1]. The discrepancies in results are most probably a consequence of differences in study design, the variation in natural light exposure related to the two study locations (Antarctica and Central Europe), and the different cognitive performance tests (Digital Symbol Substitution Test and Single Letter Cancellation Test were used in the study by Corbett et al. [1]). Also, in contrast to our study, 1-hour morning bright light application (1000 lux) resulted in shorter reaction times on the psychomotor vigilance task compared to an illuminance of 200 lux [6]. Moreover, compared to $200 \mathrm{~lx}$, after exposure to $1000 \mathrm{lux}$, application participants felt less sleepy and more energetic. The authors concluded that morning bright light can improve feelings of alertness and vitality, objective performance, and physiological arousals. In a similar setting, the same research group demonstrated significant improvements under 1700 lux vs. 165 lux (each $90 \mathrm{~min}$ exposure time) in difficult but not easy task performances (Backwards Digital-Span-Task, BDST) and no differences in the Psychomotor Vigilance Test (PV) [10].

An increase in reaction latency was posited to be responsible for the elimination of labyrinth performance after BL exposure in a mouse study [41], which could explain the lack of effect of BL on motor performance seen in our study. The decrease in performance was associated with higher levels of corticosterone in blood serum and a higher level of thigmotaxis (behavioral anxiety measure), which were also highly negatively correlated with learning and memory performance. Our group recently reported similar behaviour patterns in humans, who showed impaired sustained attention after short morning BL exposure [20].

Most recent investigations of BL and physical performance focused on endurance and strength-specific performance, showing an enhancing effect of BL [25, 26]. Early investigations reported that, in contrast to 50 lux light exposure, the application of 5000 lux is sufficient to increase handgrip strength, associated with lower rectal temperatures [42]. Suppression of melatonin and associated thermoregulatory changes through BL exposure demonstrates the potential of a "pre-cooling" effect prior to long-lasting endurance exercise [23, 26, 42]. A concomitant increase in individual strain is thought to be an important determining factor in physical performance enhancement as a result of BL exposure [25]. In contrast, in a randomized controlled trial, BL exposure $17 \mathrm{~h}$ after the individual midpoint of sleep reduced melatonin levels but failed to improve reaction time or handgrip strength in athletes [22]. In the same setting, 
evening light exposure enabled top athletes to better maintain performance across a 12-min cycling time trial [43]. Nevertheless, our results indicate a limited effect of $\mathrm{BL}$ on sensorimotor skills. In addition to the variations in the circadian phase shift that result from light exposures at different times of the day, the type of physical performance must be taken into consideration; strength and endurance performances are primarily dependent on muscular and cardiovascular parameters, whereas the nervous system predominantly determines visuomotor and sensorimotor performance.

O'Brien and O'Connor [44] found that heart rate, power capacity, and perceived exertion, as well as the oxygen consumption, alertness, and mood of competitive cyclists during an incremental exercise test, were identical in three different light intensities. Performance output measures for supra-maximum intensities were also unaltered in long-distance runners [45], even though higher light intensity reduced glucose and adrenaline levels. This evidence, as well as the present results, leads to the presumption that, with respect to light environment, the human organism reacts in a similar way when it comes to coordinative, cognitive, and endurance performance.

The suggestion that the chronotype of an individual must also be considered when the effect of $\mathrm{BL}$ on physical performance is evaluated [25] could only partially be confirmed with the present data (i.e., changes in aMT6s/ crea levels from pre- to post-light exposure). In contrast to those previous reports, the chronotype differences in the present study were between the moderate and evening chronotype. The low number of participants and the different study designs ( $2 \mathrm{~h}$ light exposure in the evening/night) may account for the lack of light effect on the remaining parameters. Since the performance tests and the urine collections were done at fixed time points in our study, influences of the individual circadian phases on the absolute values of aMT6 have to be considered. For example, for a volunteer with an eveningchronotype, a wake-up time of 6 a.m. is very early and may have negatively influenced the performance tests.

The BL exposure time might be also a factor contributing to BL-induced changes in performance parameters.

\section{Biochemical data and their association with performance}

The hypothesis of augmented suppression of aMT6s after BL exposure, along with enhanced visuomotor reaction times, cannot be confirmed with the present data. The time course of aMT6s/crea and the missing differences of aMT6s/crea between BL and OL were similar in the same setup by measuring serum melatonin concentrations [20]. Contrary to this, Chellappa et al. [18] found strong correlations between faster reaction times and salivary melatonin concentrations. It was also recently shown [46] that a regimen comprising a trp- enriched diet in combination with BL exposure during the day promoted melatonin secretion at night.

Even if the aMT6s courses did not show any associations with performance parameters in the present study, correlations between kyn and visuomotor performance parameters were found. These findings indicate that the kyn pathway of trp degradation could influence certain aspects of visuomotor performance, emphasizing the potential and importance of their implementation in future research on $\mathrm{BL}$ and performance. Trp is to a minor degree metabolized to serotonin and subsequently converted to melatonin, while trp is degraded within the so-called kyn pathway to kyn to a major degree [40]. Up to now, only a few studies on the possible effects of plasma trp on cognitive functions and exercise performance in healthy subjects have been published. Most studies focused on trp depletion or supplementation either in healthy volunteers or in patients with psychiatric diseases. In healthy individuals, acute trp depletion reduced the perception of facial emotions during OL exposure, which was not detected during BL application [29]. A trp-enriched diet on the antecedent evening was successful in increasing morning alertness and attention in subjects with mild sleep complaints [47]. In the setting of acute and chronic diseases, however, dietary intake of trp may improve a variety of neuropsychological functions $[48,49]$. Two hours of BL exposure resulted in a small increase in subjective wellbeing (affective state) in healthy subjects who were being subjected to relatively unpleasant conditions; OL was associated with a corresponding reduction in these parameters. Under both conditions, serum trp was reduced, indicating no lightspecific effect on trp [30].

Simple and effective strategies for enhancing cognitive and physical performance are desirable in the working world, as well as in competitive sports. BL has previously been shown to have the potential to meet these demands. In the present study, we were not able to prove BL's performance-enhancing properties. Nevertheless, the evaluation of trp degradation pathways revealed some interesting information regarding the effect of a single, short BL exposure on biochemical data; the fact that trp degradation was affected by the BL regimen emphasizes that the concept of including trp and its metabolites in BL research is a desirable and appropriate approach.

\section{Limitations}

One limitation of our study was that situations outside the protocol were not controlled for light. Low intensity light is physiologically important $[50,51]$, and the possibility cannot be excluded that BL application immediate after waking would have resulted in more pronounced differences between BL and OL. Exposure time to BL might also be a factor contributing to BL-induced 
changes in performance parameters. A dose-response relationship between the duration of BL light exposure and cycling performance was recently shown [52]. Thus, we cannot exclude the possibility that a longer exposure duration than the $30 \mathrm{~min}$ of $\mathrm{BL}$ applied in our study would have resulted in more significant differences between the BL and OL experiments.

The analysis of urinary sulfatoxymelatonin (aMT6s/ crea) has some limitations since the absolute values are dependent on the time length of urine pooling, and the statistical comparison between time courses of aMT6/crea with serum kynurenine and tryptophan was not possible. However, the aMT6s/crea changes in OL and BL were similar to those found in serum melatonin in previously published data from the overall research project [20].

Elimination of a first-order effect was attempted by performing a test phase before each performance trial. Nevertheless, the observed results suggest the possibility that a light-dependent effect was potentially confounded by a training effect, substantiated by increased performance after both light exposures, which was independent of light intensity.

\section{Conclusion}

Our results demonstrate that application of bright light in the morning hours has a limited effect on visuo- and sensorimotor performance. Tryptophan degradation pathways in the morning show diverse courses after office light and bright light exposure. This suggests that tryptophan can potentially be altered by bright light exposure.

\section{Abbreviations}

ANOVA: Analysis of variance; BL: Bright light; Cl: Confidence interval; CR: Correct reaction; FR: False reaction; HPLC: High-pressure liquid chromatography; KYN: Kynurenine; LED: Light-emitting diode; MEQ: Morningness-Eveningness Questionnaire; OL: Office light; PSQI: Pittsburgh Sleep Quality Index; RTCR: Reaction time of correct reaction; RTFR: Reaction time of false reaction; SD: Standard deviation; TRP: Tryptophan; TRT: Total reaction time

\section{Acknowledgements}

We thank Dietmar Fuchs, Division of Biological Chemistry, Medical University of Innsbruck, for the analysis of a large part of the biochemical parameters.

\section{Funding}

This project was financially supported by the Austrian Research Promoting Agency (FFG 823004, project name K-Licht intermediate, P-234). Parts of the project were also funded by the "Tiroler Wissenschaftsfond, TWF".

\section{Availability of data and materials}

The datasets used and/or analyzed during the current study are available from the corresponding author on reasonable request.

\section{Authors' contributions}

The contribution of each author is as follows. WS, VL, AG, and FH presented the idea of this study, planed the method, directed the experiments, and interpreted the results. CB and MC contributed to the experiments, data analyses, and manuscript preparation. All authors read and approved the final manuscript.

\section{Ethics approval and consent to participate}

The study was carried out in accordance with the ethical standards of the 2008 Declaration of Helsinki and was approved by the Ethics Committee of the Medical University of Innsbruck (program code: UN3696 279/4.4).

\section{Competing interests}

The authors declare that they have no competing interests. The authors alone are responsible for the content and writing of this article.

\section{Publisher's Note}

Springer Nature remains neutral with regard to jurisdictional claims in published maps and institutional affiliations.

\section{Author details}

${ }^{1}$ Institute for Sports Medicine, Alpine Medicine and Health Tourism, UMIT University for Health Sciences, Medical Informatics and Technology, Eduard Wallnöfer Zentrum 1, $6060 \mathrm{Hall}$ in Tyrol, Austria. ${ }^{2}$ Tirol Kliniken GmbH, Anichstraße 35, 6020 Innsbruck, Austria. ${ }^{3}$ Department of Biomedical Computer Science and Mechatronics, Institute of Electrical and Biomedical Engineering, UMIT - University for Health Sciences, Medical Informatics and Technology, Eduard Wallnöfer Zentrum 1, 6060 Hall in Tyrol, Austria. ${ }^{4}$ Central Institute of Medical and Chemical Diagnostics, LKH - University Hospital of Innsbruck, Anichstraße 35, 6020 Innsbruck, Tyrol, Austria. ${ }^{5}$ Department of Visual Perception, Bartenbach GmbH, Rinner Strasse 14, 6071 Aldrans, Tyrol, Austria.

Received: 10 February 2018 Accepted: 16 April 2018

Published online: 23 April 2018

\section{References}

1. Corbett RW, Middleton B, Arendt J. An hour of bright white light in the early morning improves performance and advances sleep and circadian phase during the Antarctic winter. Neurosci Lett. 2012;525(2):146-51.

2. Reilly T, Waterhouse J. Sports performance: is there evidence that the body clock plays a role? Eur J Appl Physiol. 2009:106(3):321-32.

3. Cajochen C, Blatter K. Circadian and sleep-wake dependent impact on neurobehavioral function. Psychol Belg. 2004:44(1/2):59-80.

4. Goel N, Basner M, Rao H, Dinges DF. Circadian rhythms, sleep deprivation, and human performance. Prog Mol Biol Transl Sci. 2013;119:155-90.

5. Hoffmann G, Gufler V, Griesmacher A, Bartenbach C, Canazei M, Staggl S, Schobersberger W. Effects of variable lighting intensities and colour temperatures on sulphatoxymelatonin and subjective mood in an experimental office workplace. Appl Ergon. 2008;39(6):719-28.

6. Smolders KC, de Kort YA, Cluitmans PJ. A higher illuminance induces alertness even during office hours: findings on subjective measures, task performance and heart rate measures. Physiol Behav. 2012;107(1):7-16.

7. van Bommel WJ. Non-visual biological effect of lighting and the practical meaning for lighting for work. Appl Ergon. 2006:37(4):461-6.

8. Yuda E, Ogasawara H, Yoshida Y, Hayano J. Exposure to blue light during lunch break: effects on autonomic arousal and behavioral alertness. J Physiol Anthropol. 2017;36(1):30.

9. Cajochen C, Munch M, Kobialka S, Krauchi K, Steiner R, Oelhafen P, Orgul S, Wirz-Justice A. High sensitivity of human melatonin, alertness, thermoregulation, and heart rate to short wavelength light. J Clin Endocrinol Metab. 2005;90(3):1311-6.

10. Huiberts LM, Smolders KC, de Kort YA. Non-image forming effects of illuminance level: exploring parallel effects on physiological arousal and task performance. Physiol Behav. 2016;164(Pt A):129-39.

11. Revell VL, Arendt J, Terman M, Skene DJ. Short-wavelength sensitivity of the human circadian system to phase-advancing light. J Biol Rhythm. 2005;20(3):270-2.

12. Daneault V, Dumont M, Masse E, Vandewalle G, Carrier J. Light-sensitive brain pathways and aging. J Physiol Anthropol. 2016;35:9.

13. Atkinson G, Drust B, Reilly $T$, Waterhouse J. The relevance of melatonin to sports medicine and science. Sports Med. 2003:33(11):809-31.

14. Besharse JC, McMahon DG. The retina and other light-sensitive ocular clocks. J Biol Rhythm. 2016:31(3):223-43.

15. Quera Salva MA, Hartley S, Barbot F, Alvarez JC, Lofaso F, Guilleminault C. Circadian rhythms, melatonin and depression. Curr Pharm Des. 2011;17(15): 1459-70. 
16. Yates CA, Herbert J. Differential circadian rhythms in pineal and hypothalamic 5-HT induced by artificial photoperiods or melatonin. Nature. 1976;262(5565):219-20

17. Crowley SJ, Eastman Cl. Phase advancing human circadian rhythms with morning bright light, afternoon melatonin, and gradually shifted sleep: can we reduce morning bright-light duration? Sleep Med. 2015;16(2):288-97.

18. Chellappa SL, Steiner R, Blattner P, Oelhafen P, Gotz T, Cajochen C. Nonvisual effects of light on melatonin, alertness and cognitive performance: can blue-enriched light keep us alert? PLoS One. 2011;6(1):e16429.

19. Dollins $A B$, Lynch HJ, Wurtman RJ, Deng MH, Lieberman HR. Effects of illumination on human nocturnal serum melatonin levels and performance. Physiol Behav. 1993;53(1):153-60.

20. Leichtfried V, Mair-Raggautz M, Schaeffer V, Hammerer-Lercher A, Mair G Bartenbach C, Canazei M, Schobersberger W. Intense illumination in the morning hours improved mood and alertness but not mental performance. Appl Ergon. 2015;46(Pt A):54-9.

21. Kaida K, Takeda Y, Tsuzuki K. The relationship between flow, sleepiness and cognitive performance: the effects of short afternoon nap and bright light exposure. Ind Health. 2012;50(3):189-96.

22. Knaier R, Schafer J, Rossmeissl A, Klenk C, Hanssen H, Hochsmann C, Cajochen C, Schmidt-Trucksass A. Effects of bright and blue light on acoustic reaction time and maximum handgrip strength in male athletes: a randomized controlled trial. Eur J Appl Physiol. 2017;117(8):1689-96.

23. Atkinson G, Barr D, Chester N, Drust B, Gregson W, Reilly T, Waterhouse J. Bright light and thermoregulatory responses to exercise. Int J Sports Med. 2008;29(3):188-93.

24. Fraschini F, Cesarani A, Alpini D, Esposti D, Stankov BM. Melatonin influences human balance. Biol Signals Recept. 1999;8(1-2):111-9.

25. Kantermann T, Forstner S, Halle M, Schlangen L, Roenneberg T, SchmidtTrucksass A. The stimulating effect of bright light on physical performance depends on internal time. PLoS One. 2012;7(7):e40655.

26. Thompson A, Jones H, Marqueze E, Gregson W, Atkinson G. The effects of evening bright light exposure on subsequent morning exercise performance. Int J Sports Med. 2015;36(2):101-6.

27. Tulppo MP, Jurvelin H, Roivainen E, Nissila J, Hautala AJ, Kiviniemi AM, Kiviniemi VJ, Takala T. Effects of bright light treatment on psychomotor speed in athletes. Front Physiol. 2014;5:184.

28. Cervenka I, Agudelo LZ, Ruas JL. Kynurenines: tryptophan's metabolites in exercise, inflammation, and mental health. Science. 2017;357(6349). https://doi.org/10.1126/science.aaf9794.

29. aan het Rot M, Coupland N, Boivin DB, Benkelfat C, Young SN. Recognizing emotions in faces: effects of acute tryptophan depletion and bright light. J Psychopharmacol. 2009;24(10):1447-54.

30. Stemer B, Melmer A, Fuchs D, Ebenbichler C, Kemmler G, Deisenhammer EA. Bright versus dim ambient light affects subjective well-being but not serotonin-related biological factors. Psychiatry Res. 2015;229(3):1011-6.

31. Zigmond AS, Snaith RP. The hospital anxiety and depression scale. Acta Psychiatr Scand. 1983;67(6):361-70.

32. Buysse DJ, Reynolds CF 3rd, Monk TH, Berman SR, Kupfer DJ. The Pittsburgh Sleep Quality Index: a new instrument for psychiatric practice and research. Psychiatry Res. 1989;28(2):193-213.

33. Leichtfried V, Kantner-Rumplmair W, Bartenbach C, Guggenbichler H, Gothe RM, Schobersberger W. Bright light therapy: minimizing light induced side effects with an innovative light setup. Int J Psychiatry Clin Pract. 2010;14(4):309-12.

34. Lucas RJ, Peirson SN, Berson DM, Brown TM, Cooper HM, Czeisler CA, Figueiro MG, Gamlin PD, Lockley SW, O'Hagan JB, Price LL, Provencio I, Skene DJ, Brainard GC. Measuring and using light in the melanopsin age. Trends Neurosci. 2014;37(1):1-9.

35. Horne JA, Ostberg O. A self-assessment questionnaire to determine morningness-eveningness in human circadian rhythms. Int J Chronobiol. 1976:4(2):97-110.

36. Widner B, Werner ER, Schennach H, Wachter H, Fuchs D. Simultaneous measurement of serum tryptophan and kynurenine by HPLC. Clin Chem. 1997:43(12):2424-6.

37. Geisler S, Mayersbach P, Becker K, Schennach H, Fuchs D, Gostner JM. Serum tryptophan, kynurenine, phenylalanine, tyrosine and neopterin concentrations in 100 healthy blood donors. Pteridines. 2015;26(1):31.

38. Wellek S, Blettner M. On the proper use of the crossover design in clinical trials: part 18 of a series on evaluation of scientific publications. Dtsch Arztebl Int. 2012;109(15):276-81.
39. Lockley SW, Evans EE, Scheer FA, Brainard GC, Czeisler CA, Aeschbach D. Short-wavelength sensitivity for the direct effects of light on alertness, vigilance, and the waking electroencephalogram in humans. Sleep. 2006; 29(2):161-8.

40. Badawy AA. Kynurenine pathway of tryptophan metabolism: regulatory and functional aspects. Int J Tryptophan Res. 2017;10:1178646917691938.

41. Huang Y, Zhou W, Zhang Y. Bright lighting conditions during testing increase thigmotaxis and impair water maze performance in BALB/C mice. Behav Brain Res. 2012;226(1):26-31.

42. Zhang $P$, Tokura $H$. Influence of two different light intensities during daytime on endurance performance of handgrip exercise. Eur J Appl Physiol Occup Physiol. 1996;74(4):318-21.

43. Knaier R, Schafer J, Rossmeissl A, Klenk C, Hanssen H, Hochsmann C, Cajochen C, Schmidt-Trucksass A. Prime time light exposures do not seem to improve maximal physical performance in male elite athletes, but enhance end-spurt performance. Front Physiol. 2017;8:264.

44. O'Brien PM, O'Connor PJ. Effect of bright light on cycling performance. Med Sci Sports Exerc. 2000;32(2):439-47.

45. Ohkuwa $\mathrm{T}$, Itoh $\mathrm{H}$, Yamamoto $\mathrm{T}$, Yanagi $\mathrm{H}$, Yamazaki $Y$, Akimaru T. Effect of varying light intensity on maximal power production and selected metabolic variables. Arch Physiol Biochem. 2001;109(5):430-4.

46. Fukushige $\mathrm{H}$, Fukuda $Y$, Tanaka M, Inami K, Wada K, Tsumura Y, Kondo M, Harada T, Wakamura T, Morita T. Effects of tryptophan-rich breakfast and light exposure during the daytime on melatonin secretion at night. J Physiol Anthropol. 2014;33:33.

47. Markus CR, Jonkman LM, Lammers JH, Deutz NE, Messer MH, Rigtering N. Evening intake of alpha-lactalbumin increases plasma tryptophan availability and improves morning alertness and brain measures of attention. Am J Clin Nutr. 2005;81(5):1026-33.

48. Jenkins TA, Nguyen JC, Polglaze KE, Bertrand PP. Influence of tryptophan and serotonin on mood and cognition with a possible role of the gut-brain axis. Nutrients. 2016;8(1)https://doi.org/10.3390/nu8010056.

49. Lieben CK, Blokland A, Deutz NE, Jansen W, Han G, Hupperts RM. Intake of tryptophan-enriched whey protein acutely enhances recall of positive loaded words in patients with multiple sclerosis. Clin Nutr. 2018;37(1):321-8.

50. Gooley JJ, Chamberlain K, Smith KA, Khalsa SB, Rajaratnam SM, Van Reen E, Zeitzer JM, Czeisler CA, Lockley SW. Exposure to room light before bedtime suppresses melatonin onset and shortens melatonin duration in humans. J Clin Endocrinol Metab. 2011;96(3):E463-72.

51. Zeitzer JM, Dijk DJ, Kronauer R, Brown E, Czeisler C. Sensitivity of the human circadian pacemaker to nocturnal light: melatonin phase resetting and suppression. J Physiol. 2000;526(Pt 3):695-702.

52. Knaier R, Meister S, Aeschbacher T, Gemperle D, Rossmeissl A, Cajochen C, Schmidt-Trucksass A. Dose-response relationship between light exposure and cycling performance. Scand J Med Sci Sports. 2016;26(7):794-801.

\section{Ready to submit your research? Choose BMC and benefit from:}

- fast, convenient online submission

- thorough peer review by experienced researchers in your field

- rapid publication on acceptance

- support for research data, including large and complex data types

- gold Open Access which fosters wider collaboration and increased citations

- maximum visibility for your research: over $100 \mathrm{M}$ website views per year

At BMC, research is always in progress.

Learn more biomedcentral.com/submissions 